\title{
Cracking the WHIP: An Evaluation of Baseball's Hall of Fame Pitchers
}

\author{
Ryan D. Cahill ${ }^{\mathrm{a}}$, John W. Brady ${ }^{\mathrm{a}}$, Alexander W. Winch ${ }^{\mathrm{a}}$, Ryan P. Ashe ${ }^{\mathrm{a}}$, Paul M. Sommers ${ }^{\mathrm{a}}$
}

Walks plus hits per inning pitched (WHIP) is a key performance metric for pitchers in Major League Baseball (MLB). The authors analyze WHIP and its two components, walks and hits, to determine whether Hall of Fame pitcher performance has changed over time since the Hall of Fame's first induction in 1936. All inducted pitchers are divided into four roughly equal groups. The results reveal that pitchers inducted between 1936 and 1959 gave up significantly fewer walks per inning pitched while pitchers inducted between 1960 and 1979 gave up significantly more hits per inning pitched. However, in general, WHIP displays no statistically significant trend over time, as the small number of walks per inning pitched among early inductees is offset by a large number of hits given up per inning pitched among later inductees.

Keywords: Baseball Hall of Fame, WHIP, pitchers, t-test, simple regression

Is it getting easier for pitchers to get into baseball's Hall of Fame? First-year eligible ballplayers include 10-season veterans who have been retired for five years. An eligible player must receive at least 75 percent of the votes cast by the Baseball Writers' Association of America (BBWAA) to earn induction to Cooperstown. Players who do not receive the 75 percent plurality necessary for election have up to 15 years of eligibility with the BBWAA. Four years after their last year of eligibility, a ballplayer (who received at least 60 percent of the votes cast in any one year on the BBWAA ballot) becomes eligible for consideration by the Veterans Committee.

Some fans believe that the Hall has become less exclusive, that the gap between old-timers and the newest members has been widening in recent years. There is considerable debate about who belongs in Cooperstown and who does not. In this research note, we compare four different groups of Hall of Fame pitchers inducted between 1936 (the inaugural year of Hall of Fame voting) and 2019.

The number of walks and hits that a pitcher allows per inning, his WHIP, is one of the most common measures for evaluating a pitcher's performance in baseball. While a low WHIP does not guarantee one's immortalization in Cooperstown, this popular statistic assesses how well (or poorly) a pitcher keeps batters off base; the lower the WHIP, the better. Only two pitchers in Major League Baseball (MLB) history have career WHIPs under 1.000: Addie Joss (.9678, inducted in 1978 by the Veterans Committee) and Ed Walsh (.9896, inducted in 1946 by the Veterans Committee). Yankee pitching great Mariano Rivera (1.0003) became baseball's first unanimous Hall of Fame selection in 2019.

\section{The Data}

Of the eighty Hall of Fame pitchers listed at www.baseball.reference.com [1], we only include inductees whose primary position was pitcher [2]. The six excluded players (and their respective years pitching in MLB) are: Babe Ruth (1914-21, 1930, 1933); Clark Griffith (1891-1914); Hank O'Day (1884-90); Clark Cummings (1872-77); and Al Spalding (1871-78). Satchel Paige played most of his career in the Negro Leagues (before Jackie Robinson integrated the sport in 1947) and did not make his MLB debut (for the Cleveland Indians) until 1948 at the age of 42 . His 476 innings pitched is lowest among all pitchers in the Hall of Fame; Bruce Sutter's 1042 innings pitched (1976-88) is the second lowest number of innings pitched among Hall of Famers. Given his late start in MLB, we excluded Satchel Paige.

We divided our remaining seventy-three pitchers into four groups, each marking a different time period of induction into the Hall of Fame. These time periods are 1936-59

(19 pitchers), 1960-79 (22), 1980-99 (17), and 2000-19 (15).

Table 1. Statistics for Hall of Fame Pitchers

\begin{tabular}{|c|c|c|c|c|c|c|c|c|}
\hline Pitcher & $\begin{array}{c}\text { Year } \\
\text { Inducted }\end{array}$ & $\begin{array}{c}\text { Years } \\
\text { Played }\end{array}$ & WHIP & IP & $\begin{array}{c}\text { Games } \\
\text { Played }\end{array}$ & $\begin{array}{c}\text { IP per } \\
\text { Game }\end{array}$ & $\begin{array}{c}\text { Walks } \\
\text { per 9 IP }\end{array}$ & $\begin{array}{c}\text { Hits } \\
\text { per 9 IP }\end{array}$ \\
\hline Walter Johnson & 1936 & $1907-1927$ & 1.061 & 5914.1 & 802 & 7.374 & 2.074 & 7.476 \\
\hline Christy Mathewson & 1936 & $1900-1916$ & 1.058 & 4788.2 & 636 & 7.529 & 1.594 & 7.929 \\
\hline Cy Young & 1937 & $1890-1911$ & 1.130 & 7356.0 & 906 & 8.119 & 1.489 & 8.677 \\
\hline Pete Alexander & 1938 & $1911-1930$ & 1.121 & 5190.0 & 696 & 7.457 & 1.649 & 8.442 \\
\hline Old Hoss Radbourn & 1939 & $1880-1891$ & 1.149 & 4527.1 & 527 & 8.591 & 1.739 & 8.604 \\
\hline Jack Chesbro & 1946 & $1899-1909$ & 1.152 & 2896.2 & 392 & 7.389 & 2.144 & 8.224 \\
\hline Joe McGinnity & 1946 & $1899-1908$ & 1.188 & 3441.1 & 465 & 7.401 & 2.124 & 8.568 \\
\hline
\end{tabular}


Research Article (2019)

\begin{tabular}{|c|c|c|c|c|c|c|c|c|}
\hline Pitcher & $\begin{array}{c}\text { Year } \\
\text { Inducted }\end{array}$ & $\begin{array}{c}\text { Years } \\
\text { Played }\end{array}$ & WHIP & IP & $\begin{array}{l}\text { Games } \\
\text { Played }\end{array}$ & $\begin{array}{l}\text { IP per } \\
\text { Game }\end{array}$ & $\begin{array}{c}\text { Walks } \\
\text { per } 9 \text { IP }\end{array}$ & $\begin{array}{c}\text { Hits } \\
\text { per } 9 \text { IP }\end{array}$ \\
\hline Eddie Plank & 1946 & 1901-1917 & 1.119 & 4495.2 & 623 & 7.216 & 2.146 & 7.924 \\
\hline Rube Waddell & 1946 & $1897-1910$ & 1.102 & 2961.1 & 407 & 7.276 & 2.440 & 7.476 \\
\hline Ed Walsh & 1946 & 1904-1917 & 1.000 & 2964.1 & 430 & 6.894 & 1.873 & 7.123 \\
\hline Lefty Grove & 1947 & $1925-1941$ & 1.278 & 3940.2 & 616 & 6.397 & 2.711 & 8.791 \\
\hline Carl Hubbell & 1947 & $1928-1943$ & 1.166 & 3590.1 & 535 & 6.711 & 1.817 & 8.676 \\
\hline Herb Pennock & 1948 & $1912-1934$ & 1.348 & 3571.2 & 617 & 5.789 & 2.308 & 9.827 \\
\hline Mordecai Brown & 1949 & $1903-1916$ & 1.066 & 3172.1 & 481 & 6.595 & 1.909 & 7.683 \\
\hline Kid Nichols & 1949 & $1890-1906$ & 1.224 & 5067.1 & 621 & 8.160 & 2.259 & 8.754 \\
\hline Chief Bender & 1953 & $1903-1925$ & 1.113 & 3017.0 & 459 & 6.573 & 2.124 & 7.890 \\
\hline Dizzy Dean & 1953 & 1930-1947 & 1.206 & 1967.1 & 317 & 6.206 & 2.072 & 8.779 \\
\hline Ted Lyons & 1955 & $1923-1946$ & 1.348 & 4161.0 & 594 & 7.005 & 2.425 & 9.709 \\
\hline Dazzy Vance & 1955 & $1915-1935$ & 1.230 & 2966.2 & 442 & 6.712 & 2.548 & 8.522 \\
\hline Bob Feller & 1962 & $1936-1956$ & 1.316 & 3827.0 & 570 & 6.714 & 4.148 & 7.692 \\
\hline John Clarkson & 1963 & 1882-1894 & 1.209 & 4536.1 & 531 & 8.543 & 2.363 & 8.521 \\
\hline Eppa Rixey & 1963 & $1912-1933$ & 1.272 & 4494.2 & 692 & 6.495 & 2.167 & 9.277 \\
\hline Red Faber & 1964 & 1914-1933 & 1.302 & 4086.2 & 669 & 6.109 & 2.671 & 9.043 \\
\hline Burleigh Grimes & 1964 & 1916-1934 & 1.365 & 4180.0 & 616 & 6.786 & 2.788 & 9.500 \\
\hline Tim Keefe & 1964 & $1880-1893$ & 1.123 & 5049.2 & 600 & 8.416 & 2.198 & 7.910 \\
\hline Pud Galvin & 1965 & $1875-1892$ & 1.191 & 6003.1 & 705 & 8.515 & 1.117 & 9.602 \\
\hline Red Ruffing & 1967 & 1924-1947 & 1.341 & 4344.0 & 624 & 6.962 & 3.193 & 8.876 \\
\hline Stan Coveleski & 1969 & $1912-1928$ & 1.251 & 3082.0 & 450 & 6.849 & 2.342 & 8.921 \\
\hline Waite Hoyt & 1969 & $1918-1938$ & 1.340 & 3762.1 & 674 & 5.582 & 2.399 & 9.657 \\
\hline Jesse Haines & 1970 & 1918-1937 & 1.350 & 3208.2 & 555 & 5.781 & 2.443 & 9.705 \\
\hline Rube Marquard & 1971 & $1908-1925$ & 1.237 & 3306.2 & 536 & 6.169 & 2.335 & 8.799 \\
\hline Lefty Gomez & 1972 & 1930-1943 & 1.352 & 2503.0 & 368 & 6.802 & 3.937 & 8.234 \\
\hline Sandy Koufax & 1972 & $1955-1966$ & 1.106 & 2324.1 & 397 & 5.855 & 3.163 & 6.792 \\
\hline Early Wynn & 1972 & $1939-1963$ & 1.329 & 4564.0 & 691 & 6.605 & 3.500 & 8.462 \\
\hline Warren Spahn & 1973 & $1942-1965$ & 1.195 & 5243.2 & 750 & 6.992 & 2.461 & 8.290 \\
\hline Mickey Welch & 1973 & $1880-1892$ & 1.226 & 4802.0 & 565 & 8.499 & 2.431 & 8.599 \\
\hline Whitey Ford & 1974 & 1950-1967 & 1.215 & 3170.1 & 498 & 6.366 & 3.083 & 7.852 \\
\hline Bob Lemon & 1976 & 1941-1958 & 1.337 & 2850.0 & 460 & 6.196 & 3.951 & 8.081 \\
\hline Robin Roberts & 1976 & 1948-1966 & 1.170 & 4688.2 & 676 & 6.936 & 1.731 & 8.795 \\
\hline Amos Rusie & 1977 & 1989-1901 & 1.349 & 3778.2 & 463 & 8.161 & 4.066 & 8.072 \\
\hline Addie Joss & 1978 & $1902-1910$ & 0.968 & 2327.0 & 286 & 8.136 & 1.408 & 7.302 \\
\hline Bob Gibson & 1981 & $1959-1975$ & 1.188 & 3884.1 & 528 & 7.357 & 3.096 & 7.597 \\
\hline Juan Marichal & 1983 & 1960-1975 & 1.101 & 3507.0 & 471 & 7.446 & 1.820 & 8.092 \\
\hline Don Drysdale & 1984 & 1956-1969 & 1.148 & 3432.0 & 518 & 6.625 & 2.242 & 8.087 \\
\hline Hoyt Wilhelm & 1985 & $1952-1972$ & 1.125 & 2254.1 & 1070 & 2.107 & 3.106 & 7.014 \\
\hline Catfish Hunter & 1987 & $1965-1979$ & 1.134 & 3449.1 & 500 & 6.899 & 2.489 & 7.718 \\
\hline Jim Palmer & 1990 & $1965-1984$ & 1.180 & 3948.0 & 558 & 7.075 & 2.989 & 7.634 \\
\hline
\end{tabular}


Research Article (2019)

\begin{tabular}{|c|c|c|c|c|c|c|c|c|}
\hline Pitcher & $\begin{array}{c}\text { Year } \\
\text { Inducted }\end{array}$ & $\begin{array}{c}\text { Years } \\
\text { Played }\end{array}$ & WHIP & IP & $\begin{array}{l}\text { Games } \\
\text { Played }\end{array}$ & $\begin{array}{l}\text { IP per } \\
\text { Game }\end{array}$ & $\begin{array}{c}\text { Walks } \\
\text { per } 9 \text { IP }\end{array}$ & $\begin{array}{c}\text { Hits } \\
\text { per } 9 \text { IP }\end{array}$ \\
\hline Fergie Jenkins & 1991 & $1965-1983$ & 1.142 & 4500.2 & 664 & 6.778 & 1.994 & 8.283 \\
\hline Gaylord Perry & 1991 & $1962-1983$ & 1.181 & 5350.0 & 777 & 6.885 & 2.320 & 8.307 \\
\hline Rollie Fingers & 1992 & $1968-1985$ & 1.156 & 1701.1 & 944 & 1.802 & 2.603 & 7.797 \\
\hline Hal Newhouser & 1992 & $1939-1955$ & 1.311 & 2993.0 & 488 & 6.133 & 3.756 & 8.041 \\
\hline Tom Seaver & 1992 & $1967-1986$ & 1.121 & 4783.0 & 656 & 7.291 & 2.616 & 7.472 \\
\hline Steve Carlton & 1994 & $1965-1988$ & 1.247 & 5217.2 & 741 & 7.041 & 3.162 & 8.059 \\
\hline Vic Willis & 1995 & $1898-1910$ & 1.209 & 3996.0 & 513 & 7.789 & 2.730 & 8.155 \\
\hline Jim Bunning & 1996 & $1955-1971$ & 1.179 & 3760.1 & 591 & 6.363 & 2.393 & 8.217 \\
\hline Phil Niekro & 1997 & 1964-1987 & 1.268 & 5404.0 & 864 & 6.255 & 3.013 & 8.400 \\
\hline Don Sutton & 1998 & 1966-1988 & 1.142 & 5282.1 & 774 & 6.825 & 2.288 & 7.994 \\
\hline Nolan Ryan & 1999 & 1966-1993 & 1.247 & 5386.0 & 807 & 6.674 & 4.670 & 6.555 \\
\hline Dennis Eckersley & 2004 & $1975-1998$ & 1.161 & 3285.2 & 1071 & 3.068 & 2.022 & 8.426 \\
\hline Bruce Sutter & 2006 & 1976-1988 & 1.140 & 1042.0 & 661 & 1.576 & 2.669 & 7.592 \\
\hline Rich Gossage & 2008 & 1972-1994 & 1.232 & 1809.1 & 1002 & 1.806 & 3.641 & 7.446 \\
\hline Bert Blyleven & 2011 & 1970-1992 & 1.198 & 4970.0 & 692 & 7.182 & 2.394 & 8.388 \\
\hline Tom Glavine & 2014 & $1987-2008$ & 1.314 & 4413.1 & 682 & 6.471 & 3.059 & 8.765 \\
\hline Greg Maddux & 2014 & 1986-2008 & 1.143 & 5008.1 & 744 & 6.732 & 1.795 & 8.493 \\
\hline Randy Johnson & 2015 & $1988-2009$ & 1.171 & 4135.1 & 618 & 6.691 & 3.258 & 7.282 \\
\hline Pedro Martinez & 2015 & $1992-2009$ & 1.054 & 2827.1 & 476 & 5.940 & 2.419 & 7.070 \\
\hline John Smoltz & 2015 & $1988-2009$ & 1.176 & 3473.0 & 723 & 4.804 & 2.617 & 7.966 \\
\hline Trevor Hoffman & 2018 & $1993-2010$ & 1.058 & 1089.1 & 1035 & 1.052 & 2.536 & 6.990 \\
\hline Jack Morris & 2018 & 1977-1994 & 1.296 & 3824.0 & 549 & 6.965 & 3.271 & 8.395 \\
\hline Roy Halladay & 2019 & 1998-2013 & 1.178 & 2749.1 & 416 & 6.609 & 1.938 & 8.662 \\
\hline Mike Mussina & 2019 & 1991-2008 & 1.192 & 3562.2 & 537 & 6.634 & 1.983 & 8.741 \\
\hline Mariano Rivera & 2019 & $1995-2013$ & 1.000 & 1283.2 & 1115 & 1.151 & 2.005 & 6.997 \\
\hline Lee Smith & 2019 & 1980-1997 & 1.256 & 1289.1 & 1022 & 1.262 & 3.392 & 7.909 \\
\hline
\end{tabular}

www.baseball-reference.com

Table 1 lists each of the seventy-three pitchers, their name as it appears on www.baseball-reference.com, the year they were inducted, the years they played in MLB, their career WHIP, career innings pitched (IP), games played, IP per game, walks per 9 IP, and hits per 9 IP. Insofar as IP is concerned, ". 1 " refers to one-third of an inning and " 2 " refers to two-thirds of an inning. To calculate walks and hits per nine innings pitched, ".1" and ".2" are converted to their decimal equivalents (.333 and .667 , respectively).

\section{Methodology}

We conducted six two-sample $t$-tests in order to compare the mean WHIP in one time period to that of every other time period. The null hypothesis states that the mean WHIP in one time period is equal to that of another time period. The twotailed alternative hypothesis states that the two mean WHIPs are not equal. In addition, we ran a simple regression of career WHIP against the induction year. We also regressed the two components of WHIP, hits per nine innings pitched and walks per nine innings pitched, against the induction year. 
Research Article (2019)

The Results

Table 2. Differences in Average WHIPs among Hall of Fame Pitchers

\begin{tabular}{|c|c|c|c|c|}
\hline \multicolumn{2}{|c|}{ Years Inducted } & \multicolumn{2}{|c|}{ Average WHIP } & \multirow{2}{*}{$p$-value on difference } \\
\hline Group 1 & Group 2 & Group 1 & Group 2 & \\
\hline $\begin{array}{c}1936-1959 \\
(n=19)\end{array}$ & $\begin{array}{c}1960-1979 \\
(n=22)\end{array}$ & 1.161 & 1.252 & 0.0052 \\
\hline $\begin{array}{c}1936-1959 \\
(n=19)\end{array}$ & $\begin{array}{c}1980-1999 \\
(n=17)\end{array}$ & 1.161 & 1.181 & 0.4540 \\
\hline $\begin{array}{c}1936-1959 \\
(n=19)\end{array}$ & $\begin{array}{c}2000-2019 \\
(n=15)\end{array}$ & 1.161 & 1.171 & 0.7469 \\
\hline $\begin{array}{c}1960-1979 \\
(n=22)\end{array}$ & $\begin{array}{c}1980-1999 \\
(n=17)\end{array}$ & 1.252 & 1.181 & 0.0140 \\
\hline $\begin{array}{c}1960-1979 \\
(n=22)\end{array}$ & $\begin{array}{c}2000-2019 \\
(n=15)\end{array}$ & 1.252 & 1.171 & 0.0164 \\
\hline $\begin{array}{c}1980-1999 \\
(n=17)\end{array}$ & $\begin{array}{c}2000-2019 \\
(n=15)\end{array}$ & 1.181 & 1.171 & 0.7060 \\
\hline
\end{tabular}

${ }^{1} \mathrm{All} p$-values in boldface are significant at better than the .05 level for a two-tailed test.

Table 2 summarizes the results of the six $t$-tests that compare the mean WHIP of Hall of Fame pitchers across different time periods. Three of the six tests revealed a discernible difference in average career WHIPs $(\alpha=.05)$ : $1936-59$ vs. $1960-79$ ( $p=.0052), 1960-79$ vs. $1980-99(p=$ $.014)$, and 1960-79 vs. 2000-19 $(p=.0164)$. All three significant differences involve pitchers inducted between 1960 and 1979. Their mean career WHIP was higher than that of any other group of inductees.

The regression of career WHIP against the induction year is shown in equation (1) ( $t$-values in parentheses):

(1)

$$
\text { WHIP }=\begin{aligned}
& 1.2275- \\
& (1.40) \quad\left(-00002 y e a r_{-}\right. \text {inducted } \\
& \mathrm{R}^{2}=<.0001
\end{aligned}
$$

Equation (1) shows that despite a higher average WHIP among pitchers inducted between 1960 and 1979, there is no discernable time trend over the last eighty-three years.

Table 3. Differences in Average Walks per 9 IP among Hall of Fame Pitchers

\begin{tabular}{|c|c|c|c|c|}
\hline \multicolumn{2}{|c|}{ Years Inducted } & \multicolumn{2}{c|}{ Average Walks per 9 IP } & \multirow{2}{*}{ p-value on difference $^{\text {1 }}$} \\
\hline $\begin{array}{c}\text { Group 1 } \\
\begin{array}{c}1936-1959 \\
(\mathrm{n}=19)\end{array}\end{array}$ & $\begin{array}{c}1960-1979 \\
(\mathrm{n}=22)\end{array}$ & 2.076 & 2.723 & $\mathbf{0 . 0 0 3 0}$ \\
\hline $\begin{array}{c}1936-1959 \\
(\mathrm{n}=19)\end{array}$ & $\begin{array}{c}1980-1999 \\
(\mathrm{n}=17)\end{array}$ & 2.076 & 2.782 & $\mathbf{0 . 0 0 0 3}$ \\
\hline $\begin{array}{c}1936-1959 \\
(\mathrm{n}=19)\end{array}$ & $\begin{array}{c}2000-2019 \\
(\mathrm{n}=15)\end{array}$ & 2.076 & 2.600 & $\mathbf{0 . 0 0 2 8}$ \\
\hline $\begin{array}{c}1960-1979 \\
(\mathrm{n}=22)\end{array}$ & $\begin{array}{c}1980-1999 \\
(\mathrm{n}=17)\end{array}$ & 2.723 & 2.782 & 0.8144 \\
\hline $\begin{array}{c}1960-1979 \\
(\mathrm{n}=22)\end{array}$ & $\begin{array}{c}2000-2019 \\
(\mathrm{n}=15)\end{array}$ & 2.723 & 2.600 & 0.6283 \\
\hline $\begin{array}{c}1980-1999 \\
(\mathrm{n}=17)\end{array}$ & $\begin{array}{c}2000-2019 \\
(\mathrm{n}=15)\end{array}$ & 2.782 & 2.600 & 0.4349 \\
\hline
\end{tabular}

\footnotetext{
${ }^{1} \mathrm{All} p$-values in boldface are significant at better than the .05 level for a two-tailed test.
} 
Table 3 shows the results of the $t$-tests comparing average walks per nine innings pitched across all four time periods. The three tests that yield significant differences all involve the time period 1936-59. In particular, pitchers inducted between 1936 and 1959 gave up significantly fewer walks per nine innings pitched, on average.

Table 4. Differences in Average Hits per 9 IP among Hall of Fame Pitchers

\begin{tabular}{|c|c|c|c|c|}
\hline \multicolumn{2}{|c|}{ Year Inducted } & \multicolumn{2}{c|}{ Aver age Hits per 9 IP } & \multirow{2}{*}{ p-value on difference $^{\mathbf{l}}$} \\
\hline Group 1 & Gr oup 2 & Group 1 & Group 2 & 0.4642 \\
\hline $\begin{array}{c}1936-1959 \\
(\mathrm{n}=19)\end{array}$ & $\begin{array}{c}1960-1979 \\
(\mathrm{n}=22)\end{array}$ & 8.372 & 8.545 & $\mathbf{0 . 0 1 5 1}$ \\
\hline $\begin{array}{c}1936-1959 \\
(\mathrm{n}=19)\end{array}$ & $\begin{array}{c}1980-1999 \\
(\mathrm{n}=17)\end{array}$ & 8.372 & 7.848 & 0.0790 \\
\hline $\begin{array}{c}1936-1959 \\
(\mathrm{n}=19)\end{array}$ & $\begin{array}{c}2000-2019 \\
(\mathrm{n}=15)\end{array}$ & 8.372 & 7.941 & $\mathbf{0 . 0 0 2 5}$ \\
\hline $\begin{array}{c}1960-1979 \\
(\mathrm{n}=22)\end{array}$ & $\begin{array}{c}1980-1999 \\
(\mathrm{n}=17)\end{array}$ & 8.545 & 7.848 & $\mathbf{0 . 0 1 8 9}$ \\
\hline $\begin{array}{c}1960-1979 \\
(\mathrm{n}=22)\end{array}$ & $\begin{array}{c}2000-2019 \\
(\mathrm{n}=15)\end{array}$ & 8.545 & 7.941 & 0.6511 \\
\hline $\begin{array}{c}1980-1999 \\
(\mathrm{n}=17)\end{array}$ & $\begin{array}{c}2000-2019 \\
(\mathrm{n}=15)\end{array}$ & 7.848 & 7.941 & 0 \\
\hline
\end{tabular}

${ }^{1} \mathrm{All} p$-values in boldface are significant at better than the .05 level for a two-tailed test.

Table 4 shows the results of the $t$-tests comparing average hits per nine innings pitched across different time periods. The statistically significant tests include 1936-59 vs. 1980-99 $(p=.0151), 1960-79$ vs. $1980-99(p=.0025)$, and $1960-79$ vs. 2000-19 ( $p=.0189)$. For two of these three tests, pitchers inducted into the Hall between 1960 and 1979, on average, gave up significantly more hits per nine innings pitched than pitchers of any other time period, except for the first time period. This result explains why these same pitchers (inducted between 1960 and 1979) had significantly higher average WHIPs than any other group.

The results in Tables 3 and 4 suggest that walks per nine innings pitched are trending up over time $(p=.008)$, while hits given up per nine innings pitched are trending down over time $(p=.01)$. We regressed each of these two primary components of WHIP against induction year, as shown in equations (2) and (3) ( $t$-values in parentheses):

$$
\begin{aligned}
& \text { walks_per_9_IP }=-14.2199+.0085 y e a r_{-} \text {inducted } \\
& \begin{array}{lll}
(-2.30) \quad(2.71) \quad \mathrm{R}^{2} & =.0936
\end{array}
\end{aligned}
$$

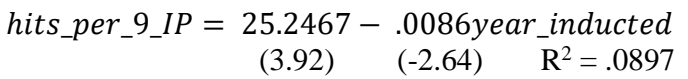

Interestingly, the regressions indicate that over time, the number of walks given up per nine innings pitched have increased while the number of hits given up per nine innings pitched have decreased. The slope coefficients in equations (2) and (3) are practically equal and opposite in sign and explain why WHIP has been relatively constant over time.

\section{Concluding Remarks}

Career WHIPs among Hall of Fame pitchers have changed very little since the first year of balloting in 1936, although recent inductees have given up more walks but fewer hits per nine innings pitched. The evidence presented here may not settle the question whether a ticket to Cooperstown is getting any cheaper for pitchers. But, in an era marked by pitch counts and early exits, the career WHIPs of recently inducted pitchers (more of whom are relievers) match up well with pitchers already in the Hall of Fame. 
Research Article (2019)

\section{References}

1. "MLB Stats, Scores, History, \& Records." BR Bullpen, www.baseball-reference.com/.
2. "Hall of Fame Explorer." Baseball Hall of Fame, National Baseball Hall of Fame and Museum, www.baseballhall.org/. 